\title{
THE ADMISSIBLE MEAN VALUES OF A STOCHASTIC PROCESS
}

BY

T. S. PITCHER

If $x(t)$ is a real valued stochastic process on the linear set $T$ and $f$ is a function on $T$, we will write $P_{x}$ and $P_{x+f}$ for the probability measures induced on sample space by the processes $x(t)$ and $x(t)+f(t)$ respectively. Thus, if $g$ is a measurable function of $x\left(t_{1}\right), \cdots, x\left(t_{n}\right)$,

$$
\int g\left(x\left(t_{1}\right), \cdots, x\left(t_{n}\right)\right) P_{x+f}(d x)=\int g\left(x\left(t_{1}\right)+f\left(t_{1}\right), \cdots, x\left(t_{n}\right)+f\left(t_{n}\right)\right) P_{x}(d x) .
$$

We will say that $f$ is an admissible mean value for $x$ over the field $S$ if $P_{x+f}$ is absolutely continuous with respect to $P_{x}$ (which we will hereafter write $P_{x+f}<P_{x}$ ) over $S$. The purpose of this paper is to investigate the size and structure of $M(x, S)$, the set of admissible mean values.

The field of most interest and the one generally referred to in this paper is that generated by all the random variables $x(t)$ for $t$ in $T$. We will write $S^{\prime}$ for this field but will often suppress it in the notation and when no field is explicitly mentioned $S^{\prime}$ is meant. All the fields considered will be subfields of $S^{\prime}$. A knowledge of $M(x, S)$ is important in setting up "signal plus noise" problems (i.e., problems of discriminating between $x$ and $x+f$ ) since a realistic set of assumptions should usually lead to a set of $f$ 's contained in $M(x, S)$ for the relevant $S$.

I. The size of $M(x)$ in the $L_{2}$ case. Throughout this section we will assume that $x(t)$ is a measurable process, that $T$ is a finite or infinite interval, that $\int x(t) P_{x}(d x)=0$ and $\int x^{2}(t) P_{x}(d x)<\infty$ for all $t$, and that $\int_{T} x^{2}(t) d t<\infty$ with probability one. The assumption that the mean value vanishes is harmless since, as is easily seen, $P_{x+g+f}<P_{x+g}$ if and only if $P_{x+f}<P_{x}$. We will also assume that the correlation function $R(s, t)=\int x(s) x(t) P_{x}(d x)$ satisfies $\int_{T} \int_{T} R^{2}(s, t) d s d t<\infty$ so that the integral transform $R: R f(s)=\int_{T} R(s, t) f(t) d t$ is a compact operator on $L_{2}(T)$.

EXAMPLE 1. If $x(t)$ is a Gaussian process with continuous sample functions, then $M(x)=R^{1 / 2}\left(L_{2}(T)\right.$ ) (where for each $f$ in $R^{1 / 2}\left(L_{2}(t)\right.$ ) its continuous version is chosen). This is proved in [2]. Theorem 1 below shows that this is as large as $M(x)$ can get.

Example 2. If $x(t)$ is a Poisson process, then any $f$ in $M(x)$ must be a jump function and since the probability of a jump at any point is zero, $M(x)=(0)$.

Received by the editors August 8, 1962. 
EXAMPLE $3\left({ }^{1}\right)$. Let $x(t)$ be a process with independent increments whose Levy representation is

$$
\begin{aligned}
\log \int e^{i \lambda x(t)} P_{x}(d x)= & -\frac{1}{2} \sigma^{2}(t) \lambda^{2}+\int_{|u| \leqq 1 ; 0 \leqq \tau \leqq t}\left(e^{i \lambda u}-1-i \lambda u\right) Q(d \tau, d u) \\
& +\int_{|u|>1 ; 0 \leqq \imath \leqq t}\left(e^{i \lambda u}-1\right) Q(d \tau, d u) .
\end{aligned}
$$

$\sigma^{2}(t)$ is nondecreasing and represents the Gaussian part of $x(t)$, that is, we can write $x(t)=x_{G}(t)+y(t)$ where $x_{G}(t)$ is a Gaussian process with correlation function $R(s, t)=\sigma^{2}(\min (s, t))$ and $y(t)$ is independent of $x_{G}(t)$. Then, [6, Theorem 4, p. 421], $M(x)=M\left(x_{G}\right)=R^{1 / 2}\left(L_{2}(T)\right)$.

THEOREM 1. $M(x) \subset R^{1 / 2}\left(L_{2}(T)\right)$.

Proof. $x(t)$ is a measurable process and its sample functions are in $L_{2}(T)$ with probability one so, for any $h$ in $L_{2}(T), \theta(h, x)=\int_{T} x(t) h(t) d t$ is a $P_{x}$-measurable function of $x$. An easy computation shows that $\int \theta^{2}(h, x) P_{x}(d x)=\int_{T}\left(R^{1 / 2} h\right)^{2}(t) d t$. Hence, if $R^{1 / 2} h_{n}$ converges to $R^{1 / 2} h, \theta\left(h_{n}, x\right)$ is $L_{2}\left(P_{x}\right)$ convergent and some subsequence converges to $\theta(h, x)$ almost everywhere. Now the distribution of $\theta\left(h_{n}, x\right)$ with respect to $P_{x+f}$ is the same as the distribution of $\theta\left(h_{n}, x\right)+\int_{T} f(t) h_{n}(t) d t$ with respect to $P_{x}$, so if $P_{x+f}<P_{x}, \int_{T} f(t) h_{n}(t) d t$ must converge to $\int_{T} f(t) h(t) d t$ whenever $R^{1 / 2} h_{n}$ converges to $R^{1 / 2} h$, and this implies that $f$ is in $R^{1 / 2}\left(L_{2}(T)\right)$.

If $M(x)$ is a measurable set, then $P_{x}(M(x))$ would give another, more intrinsic measure of the size of $M(x)$. The only result of this kind known to the author, except for cases where $M(x)=(0)$, is in the Gaussian case where $P_{x}(M(x))=1$ or 0 depending on whether $R$ has finitely or infinitely many nonzero eigenvalues $[4$, p. 42].

II. The semigroup of isometries associated with $M(x)$. We now replace the assumptions of the previous section by a separability assumption. $x(t)$ is measurably separable if there exists a countable subset $\left(t_{i}\right)$ of $T$ such that the smallest $\sigma$ field with respect to which all the $x\left(t_{i}\right)$ are measurable is equivalent to $S^{\prime}$ under $P_{x}$. Any process whose sample functions are continuous with probability one is measurably separable. We shall write $x_{i}$ for $x\left(t_{i}\right)$ throughout this section and $S_{n}$ for the field generated by $x_{1}, \cdots, x_{n}$. If $\pi_{n} \phi$ is the conditional expectation of $\phi$ on $S_{n}$, then $\pi_{n}$ has a unique extension to a projection in $L_{p}\left(P_{x}\right)$ whenever $\infty>p \geqq 1$ and $\pi_{n}$ converges strongly to the identity. It follows that $L_{p}\left(P_{x}\right)$ is separable for $\infty>p \geqq 1$.

If $f$ is in $M(x)$ and $\phi$ is a bounded $S_{n}$-measurable function, we define for each $\infty>p \geqq 1$

(1) Another example related to this, but not of $L_{2}$ type, is afforded by the symmetric stable processes of index $\alpha<1$. Here again $M(x)=0$ since for every $t_{0}$ in $T$ and $\beta>\alpha$, $\left(t-t_{0}\right)^{-1 / \beta}\left(x(t)-x\left(t_{0}\right)\right) \rightarrow 0$ with probability one [1, Theorem 3.1, p. 497]. 


$$
u_{f}^{p} \phi(x)=\left[\frac{d P_{x+f}}{d P_{x}}\right]^{1 / p} \phi\left(x_{1}-f_{1}, \cdots, x_{n}-f_{n}\right)
$$

(writing $f_{i}$ for $f\left(t_{i}\right)$ ). $u_{f}^{p}$ is densely defined and preserves positivity and norm in $L_{p}\left(P_{x}\right)$, hence has a unique extension to a positivity preserving isometry in $L_{p}\left(P_{x}\right)$ which we also denote by $u_{f}^{p}$. This definition is clearly independent of the particular sequence $\left(t_{i}\right)$ which was used.

THEOREM 2. If $x(t)$ is measurably separable, then $f+g$ is in $M(x)$ whenever $f$ and $g$ are, and $u_{f+g}^{p}=u_{f}^{p} u_{g}^{p}$ for all $p$. The semigroup $U_{p}(x)=\left[u_{f}^{p} \mid f \in M(x)\right]$ is strongly closed.

Proof. Let $\phi$ be a bounded $S_{n}$-measurable function and $\psi$ be a nonnegative bounded $S_{m}$-measurable function. Then if $f$ is in $M(x), \int \phi(x)\left[u_{f}^{p}(\psi)(x)\right]^{p} P_{x}(d x)$ $=\int \phi(x+f)[\psi(x)]^{p} P_{x}(d x)$ and this relation extends by continuity to all nonnegative $\psi$ in $L_{p}\left(P_{x}\right)$. In particular, if $g$ is in $M(x)$. we can apply this equation with $\psi=d P_{x+g} / d P_{x}$ and $p=1$ to get

$\int \phi(x) u_{f}^{1}\left(\frac{d P_{x}+g}{d P_{x}}\right)(x) P_{x}(d x)=\int \phi(x+f) \frac{d P_{x+g}}{d P_{x}}(x) P_{x}(d x)=\int \phi(x+f+g) P_{x}(d x)$ which proves that $f+g$ is in $M(x)$ and that $d P_{x+f+g} / d P_{x}=u_{f}^{1}\left(d P_{x+g} / d P_{x}\right)$. Now applying the equation to $u_{g}^{p}(\psi)$ we have

$$
\begin{aligned}
\int \phi(x)\left[u_{f}^{p}\left(u_{g}^{p}(\psi)\right)(x)\right]^{p} P_{x}(d x)=\int \phi(x+f)\left[u_{g}^{p}(\psi)(x)\right]^{p} P_{x}(d x) \\
=\int \phi(x+f+g)[\psi(x)]^{p} P_{x}(d x)=\int \phi(x)\left[\left[u_{f+g}^{p}(\psi)(x)\right]^{p} P_{x}(d x)\right.
\end{aligned}
$$

which shows that $u_{f}^{p} u_{g}^{p}$ and $u_{f+g}^{p}$ agree on nonnegative functions and hence on all functions in $L_{p}\left(P_{x}\right)$.

Now suppose $V$ is in the strong closure of $U_{p}(x)$. For each $t$ in $T$ and real number $\lambda$, there is a sequence $\left(f_{n}\right)$ from $M(x)$ with $V(1)=\lim u_{f_{n}}^{p}(1)$ and

$$
\begin{aligned}
V(\exp i \lambda x(t)) & =\lim u_{f_{n}}^{p}(\exp i \lambda x(t))=\lim u_{f_{n}}^{p}(1) \exp i \lambda\left(x(t)+f_{n}(t)\right) \\
& =\lim V(1) \exp i \lambda\left(x(t)+f_{n}(t)\right)
\end{aligned}
$$

which shows that $f_{n}(t)$ converges to a limit which we call $f(t) . f(t)$ clearly does not depend on the particular sequence $\left(f_{n}\right)$ and since a common sequence $\left(f_{n}^{\prime}\right)$ can be found for both $\exp i \lambda x(t)$ and $\exp i \mu x(t)$, it does not depend on the choice of $\lambda$. For any real numbers $\lambda_{1}, \cdots, \lambda_{k}$ a sequence $\left(f_{n}\right)$ can be found so that $u_{f_{n}}^{p}$ will approximate $V$ on all the functions $1, \exp i \lambda_{1} x_{1}, \cdots, \exp i \lambda_{k} x_{k}$ and $\exp i \sum_{j=1}^{k} \lambda_{j} x_{j}$ and thus $V\left(\exp i \sum_{j=1}^{k} \lambda_{j} x_{j}\right)=V(1) \exp i \sum_{j=1}^{k} \lambda_{j}\left(x_{j}+f\left(t_{j}\right)\right)$. If $\hat{\phi}$ is any continuous function of $k$ real variables with compact support and $N$ is any positive number, there is a trigonometric function $\hat{\psi}_{N}$ with period $2 N$ such that $|\hat{\psi}-\hat{\phi}|<1 / N$ whenever $\left|x_{i}\right|<N, i=1, \cdots, k$. Setting 


$$
\phi(x)=\hat{\phi}\left(x_{1}+f\left(t_{1}\right), \cdots, x_{k}+f\left(t_{k}\right)\right) \text { and } \psi_{N}(x)=\hat{\psi}_{N}\left(x_{1}+f\left(t_{1}\right), \cdots, x_{k}+f\left(t_{k}\right)\right),
$$

we have $V \phi(x)=\lim _{N \rightarrow \infty} V \psi_{N}(x)=V(1) \hat{\phi}\left(x_{1}, \cdots, x_{k}\right)$. Hence,

$$
\begin{aligned}
\int V(1)^{p}(x) \hat{\phi}^{p}\left(x_{1}, \cdots, x_{k}\right) P_{x}(d x) & =\|V \phi\|=\|\phi\|^{p}, \\
& =\int \phi^{p}\left(x_{1}+f\left(t_{1}\right), \cdots, x_{k}+f\left(t_{k}\right)\right) P_{x}(d x) \\
& =\int \hat{\phi}^{p}\left(x_{1}, \cdots, x_{k}\right) P_{x+f}(d x)
\end{aligned}
$$

and, since functions of this form are dense in $L_{1}\left(P_{x}\right)$, this implies that $f$ is in $M(x)$ and that $V(1)^{p}=d P_{x+f} / d P_{x}$. Finally, since $V$ agrees with $u_{f}^{p}$ on a dense subset, $V=u_{f}^{p}$.

Corollary 1. If $M(x)=-M(x)$, in particular, if $P_{x}$ is symmetric about 0 , then $U_{2}(x)$ is a strongly closed group of unitary operators.

Corollary 2. Suppose that the joint distribution of $x_{1}, \cdots, x_{k}$ is given by a density $p_{k}\left(\xi_{1}, \cdots, \xi_{k}\right)$. Then if $\left(f_{n}\right)$ is a sequence from $M(x)$ such that $\lim f_{n}(t)=f(t)$ exists for all $t$ in $T$, and if

$$
\begin{aligned}
A(n, m)=\limsup _{k \rightarrow \infty} \int \cdots \int \mid p_{k}\left(\xi_{1}-f_{n}\left(t_{1}\right), \cdots, \xi_{k}-f_{n}\left(t_{k}\right)\right) & \\
& -p_{k}\left(\xi_{1}-f_{m}\left(t_{1}\right), \cdots, \xi_{k}-f_{m}\left(t_{k}\right)\right) \mid d \xi_{1} \cdots d \xi_{k}
\end{aligned}
$$

goes to zero as $\inf (n, m)$ goes to infinity, $f$ is also in $M(x)$ and $d P_{x+f} / d P_{x}$ is the $L_{1}\left(P_{x}\right)$ limit of $d P_{x+f_{n}} / d P_{x}$.

Proof. If $\hat{\phi}$ is a bounded function of $j$ real variables with bounded first and second derivatives and $\phi(x)=\hat{\phi}\left(x_{1}, \cdots, x_{j}\right)$, then

$$
\begin{aligned}
& \begin{aligned}
\left\|u_{f_{n}}^{1}(\phi)-u_{f_{m}}^{1}(\phi)\right\| & =\lim _{k \rightarrow \infty} \int \cdots \int \mid \frac{p_{k}\left(\xi_{1}-f_{n}\left(t_{1}\right), \cdots, \xi_{k}-f_{n}\left(t_{k}\right)\right)}{p_{k}\left(\xi_{1}, \cdots, \xi_{k}\right)} \hat{\phi}\left(\xi_{1}-f_{n}\left(t_{1}\right), \cdots, \xi_{k}-f_{n}\left(t_{k}\right)\right) \\
& -\frac{p_{k}\left(\xi_{1}-f_{m}\left(t_{1}\right), \cdots, \xi_{k}-f_{m}\left(t_{k}\right)\right)}{p_{k}\left(\xi_{1}, \cdots, \xi_{k}\right)} \hat{\phi}\left(\xi_{1}-f_{m}\left(t_{1}\right), \cdots, \xi_{k}-f_{m}\left(t_{k}\right)\right) \mid \\
\leqq & \cdot p_{k}\left(\xi_{1}, \cdots, \xi_{k}\right) d \xi_{1} \cdots d \xi_{k}
\end{aligned} \\
& +\iiint\left|p_{k}\left(\xi_{1}-f_{n}\left(t_{1}\right), \cdots, \xi_{k}-f_{n}\left(t_{k}\right)\right)-p_{k}\left(\xi_{1}-f_{m}\left(t_{1}\right), \cdots, \xi_{k}-f_{m}\left(t_{k}\right)\right)\right| d \xi_{1} \cdots d \xi_{k}
\end{aligned}
$$


and this goes to zero by hypothesis as $n$ and $m$ go to infinity. It follows that $u_{f_{n}}^{1}$ is strongly convergent, hence converges to some $u_{g}^{1}$ in $U_{1}(x)$ and $g(t)=\lim f_{n}(t)=f(t)$ for all $t$ in $T$.

THEOREM 3. $u_{f}^{p}$ has no eigenvectors if $f \neq 0 . U_{p}(x)$ has no almost periodic vectors.

Proof. The subspace of almost periodic vectors of an abelian semigroup of operators is spanned by its one-dimensional invariant subspaces [3, Satz 1.7.5, pp. $30 \mathrm{ff}$.] so the second assertion will follow from the first. If $u_{f}^{p}(\psi)=\lambda \psi$, then

$$
u_{f}^{p}(|\psi|)=\left|u_{f}^{p}(\psi)\right|=|\lambda||\psi|=|\psi| .
$$

Let $t$ be a point of $T$ at which $f$ does not vanish, say $f(t)>0$, and define $A_{n}$ to be the set where $n f(t) \leqq x(t)<(n+1) f(t)$.

$$
\int_{A_{n}}|\psi|^{p} d P=\int_{A_{n}}\left|u_{f}^{p}(|\psi|)\right|^{p} d P=\int_{\left[x \mid x(t)+f(t) \in A_{n}\right]}|\psi|^{p} d P=\int_{A_{n-1}}|\psi|^{p} d P
$$

so $\int_{A_{n}}|\psi|^{p} d P=c$, and since $\int|\psi|^{p} d P=\Sigma_{n} \int_{A_{n}}|\psi|^{p} d P<\infty$ we must have $c=0$ and hence, $\psi=0$.

III. One-parameter semigroups in $M(x)$. In this section we investigate various conditions under which an entire half line $[\alpha f \mid \alpha \geqq 0]$ belongs to $M(x)$. If $f$ is in $M(x)$, then, of course, so is $k f$ for every positive integer $k$. The following simple example shows that these may be the only multiples of $f$ in $M(x)$. We take $T$ to consist of a single point, i.e., the stochastic process is simply a single random variable $x$ and the possible mean values are just the real numbers. If $x$ is distributed according to the density $p$,

$$
p(\xi)= \begin{cases}0 & \text { if } \quad \xi<2 \\ 2^{-k} & \text { if } \quad 2 \leqq 2 k \leqq \xi<2 k+1 \\ 0 & \text { if } \quad 2 k+1 \leqq \xi<2 k+2\end{cases}
$$

then $M(x)$ is just the positive integers.

THEOREM 4. If $x(t)$ is a measurably separable process and af is in $M(x)$ for all $\alpha \geqq 0$, then $u_{\alpha f}^{p}$ is a strongly continuous semigroup.

Proof. It will be sufficient to prove the theorem for $p=1$, since

$$
\begin{aligned}
\int\left|u_{\alpha f}^{p}(\phi)(x)-\phi(x)\right|^{p} P_{x}(d x) & \leqq \int\left|\left[u_{\alpha f}^{p}(\phi)\right]^{p}(x)-\phi^{p}(x)\right| P_{x}(d x) \\
& =\int\left|u_{\alpha f}^{1}\left(\phi^{p}\right)(x)-\phi^{p}(x)\right| P_{x}(d x)
\end{aligned}
$$


for nonnegative, bounded $S_{n}$-measurable $\phi$ and differences of such functions are dense in $L_{p}\left(P_{x}\right)$. For bounded $S_{n}$-measurable $\phi$ and arbitrary $\psi$ in $L_{1}\left(P_{x}\right)$,

$$
\int \phi(x) u_{\alpha f}^{1}(\psi)(x) P_{x}(d x)=\int \phi(x+\alpha f) \psi(x) P_{x}(d x)
$$

which is a measurable function of $\alpha$ and for any bounded $\phi$,

$$
\int \phi(x) u_{\alpha f}^{1}(\psi)(x) P_{x}(d x)=\lim \int \pi_{n} \phi(x) u_{\alpha f}^{1}(\psi)(x) P_{x}(d x)
$$

so $u_{\alpha f}^{1}$ is weakly measurable. This fact plus the separability of $L_{1}\left(P_{x}\right)$ implies that $u_{\alpha f}^{1}$ is strongly continuous for $\alpha>0$ so that

$$
\lim _{\alpha \rightarrow 0}\left\|u_{\alpha f}^{1}(\psi)-\psi\right\|=\lim _{\alpha \rightarrow 0}\left\|u_{\alpha f+f}^{1}(\psi)-u_{f}^{1}(\psi)\right\|=0 .
$$

The next theorem gives the form of the infinitesimal generators of these groups in some cases. We restrict attention to the case $p=1$ because it is easier computationally and also because it is the most important in practice (see [5, Theorem 3.1, p. 15]. Let $D_{0}$ be the set of functions $\phi$ of the form $\phi(x)=\hat{\phi}\left(x_{1}, \cdots, x_{n}\right)$ where $\hat{\phi}$ is bounded and has bounded first derivatives. For $\phi$ in $D_{0}$ we define

$$
\mathscr{D} \phi(x)=\left.\frac{\partial}{\partial \alpha} \hat{\phi}\left(x_{1}+\alpha f_{1}, \cdots, x_{n}+\alpha f_{n}\right)\right|_{\alpha=0} .
$$

THEOREM 5. If $\alpha f$ is in $M(x)$ for all $\alpha \geqq 0, u_{\alpha f}^{1}$ is strongly continuous and the function 1 is in the domain of its generator $A$, then for every $\phi$ in $D_{0}$

$$
\int A(1)(x) \phi(x) P_{x}(d x)=\int \mathscr{D} \phi(x) P_{x}(d x) .
$$

Moreover, $D_{0}$ is contained in the domain of $A$ and

$$
A \phi(x)=A(1)(x) \phi(x)-\mathscr{D} \phi(x) .
$$

Conversely, if a function $\lambda(x)$ exists in $L_{1}\left(P_{x}\right)$ satisfying $\int \lambda(x) \phi(x) P_{x}(d x)=$ $\int \mathscr{D} \phi(x) P_{x}(d x)$ for all $\phi$ in $D_{0}$ and if the set $\left[\lambda(x) \phi(x)-\mathscr{D} \phi(x)-a \phi(x) \mid \phi \in D_{0}\right]$ is dense in $L_{1}\left(P_{x}\right)$ for some $a \neq 0$, then $\alpha f$ is in $M(x)$ for all real $\alpha$ and the generator of $u_{x f}^{1}$ is the closure of theoperator Adefined on $D_{0} b y: A \phi(x)=\lambda(x) \phi(x)-\mathscr{D} \phi(x)$. The same conclusion holds if instead of the condition on the density of the range, $\lambda$ satisfies either

or

$$
\liminf \int_{[x \mid \lambda(x)>n]} \lambda(x) P_{x}(d x)=O\left(e^{-\varepsilon n}\right)
$$

$$
\liminf -\int_{[x \mid \lambda(x)<-n]} \lambda(x) P_{x}(d x)=O\left(e^{-\varepsilon n}\right) \text { for some } \varepsilon>0
$$


Proof. If 1 is in the domain of $A$ and $\phi$ is in $D_{0}$, then

$$
\begin{aligned}
\frac{1}{\alpha}\left(u_{\alpha f}^{1} \phi-\phi\right)(x) & =\frac{1}{\alpha}\left(u_{\alpha f}^{1}(1)-1\right)(x) \phi(x) \\
+ & \frac{1}{\alpha}\left(u_{\alpha f}^{1}(1)-1\right)(\hat{\phi}(x-\alpha f)-\hat{\phi}(x))+\frac{1}{\alpha}(\hat{\phi}(x-\alpha f)-\hat{\phi}(x))
\end{aligned}
$$

which converges to $A(1) \phi-\mathscr{D} \phi$. Also,

$$
\begin{aligned}
\int A(1)(x) \phi(x) P_{x}(d x) & =\lim _{\alpha \rightarrow 0} \frac{1}{\alpha} \int\left(\frac{d P_{x+\alpha f}}{d P_{x}}-1\right)(x) \phi(x) P_{x}(d x) \\
& =\lim _{x \rightarrow 0} \int \frac{1}{\alpha}\left[\phi\left(x_{1}+\alpha f_{1}, \cdots, x_{n}+\alpha f_{n}\right)-\phi\left(x_{1}, \cdots, x_{n}\right)\right] P_{x}(d x) \\
& =\int \mathscr{D} \phi(x) P_{x}(d x) .
\end{aligned}
$$

The remainder of the theorem is a straightforward application of Theorems 3.3 and 3.4 of [5].

We now return to the assumptions of $\S 1$, namely, $\int x(t) P_{x}(d x)=0$, $\int x^{2}(t) P_{x}(d x)<\infty, \int_{T} x^{2}(t) d t<\infty$ with probability one, and $R(s, t)$ square integrable on $T \times T$. Let $\left(\lambda_{i}\right)$ and $\left(g_{i}\right)$ be the eigenvalues and corresponding eigenvectors of $R$. We will write $x_{n}$ for the random variables $x_{n}=\lambda_{n}^{-1 / 2} \int_{T} x(t) g_{n}(t) d t$; $S_{n}$ for the field generated by $x_{1}, \cdots, x_{n} ; S_{\infty}$ for the field generated by all $x_{n}$ 's and $\pi_{n} \phi$ for the conditional expectation of $\phi$ on $S_{n} . S_{\infty}$ is not larger than $S^{\prime}$ and for many applied problems is a more realistic field to deal with than $S^{\prime}$. Since the proof of Theorem 1 was essentially an $L_{2}$ proof, it also applies to $M\left(x, S_{\infty}\right)$ and hence the numbers $f_{n}, f_{n}=\lambda_{n}^{-1 / 2} \int_{T} f(t) g_{n}(t) d t$, satisfy $\sum_{n=1}^{\infty} f_{n}^{2}<\infty$ for any $f$ in $M\left(x, S_{\infty}\right)$.

THEOREM 6. If for every $n$ the joint distribution of $x_{1}, \cdots, x_{n}$ is given by a density $p_{n}$ satisfying

(1) $p_{n}\left(\xi_{1}, \cdots, \xi_{n}\right)>0$ almost everywhere,

(2) $\lim _{\zeta_{i} \rightarrow \pm \infty} p_{n}\left(\xi_{1}, \cdots, \xi_{j}, \cdots, \xi_{n}\right)=0$ for almost all $\xi_{1}, \cdots, \xi_{j-1}, \xi_{j+1}, \cdots, \xi_{n}$, and

(3) $\partial p_{n} / \partial \xi_{j}$ exists for all $j \leqq n$ and

$$
\int \cdots \int \frac{1}{p_{n}\left(\xi_{1}, \cdots, \xi_{n}\right)}\left(\frac{\partial p_{n}}{\partial \xi_{i}}\right)^{2}\left(\xi_{1}, \cdots, \xi_{n}\right) d \xi_{1} \cdots d \xi_{n} \leqq K,
$$

then $M\left(x, S_{\infty}\right)$ is positive linear, i.e., if $f$ is in $M\left(x, S_{\infty}\right)$, so is $\alpha f$ for all $\alpha \geqq 0$.

Proof. Assume $f$ is in $M\left(x, S_{\infty}\right) . V_{n}(\alpha)$ defined on $\pi_{n} L_{2}\left(P_{x}\right)$ by:

$$
V_{n}(\alpha) \phi\left(x_{1}, \cdots, x_{n}\right)=\left[\frac{p_{n}\left(x_{1}-\alpha f_{1}, \cdots, x_{n}-\alpha f_{n}\right)}{p_{n}\left(x_{1}, \cdots, x_{n}\right)}\right]^{1 / 2} \phi\left(x_{1}-\alpha f_{1}, \cdots, x_{n}-\alpha f_{n}\right)
$$


is a strongly continuous semigroup of isometries in $\pi_{n} L_{2}\left(P_{x}\right)$. Let $J_{n}(\lambda)$ be the resolvent of $V_{n}(\alpha)$. The lower semimartingale

$$
y_{n}(x)=\left[\frac{p_{n}\left(x_{1}-\alpha f_{1}, \cdots, x_{n}-\alpha f_{n}\right)}{p_{n}\left(x_{1}, \cdots, x_{n}\right)}\right]^{1 / 2}
$$

is uniformly in $L_{2}\left(P_{x}\right)$ and hence converges in mean to some function $Q_{\alpha}$. The operators $V(\alpha)$ defined on each $\pi_{n} L_{2}\left(P_{x}\right)$ by $V(\alpha) \phi(x)=Q_{\alpha}(x) \phi\left(x_{1}-\alpha f_{1}, \cdots, x_{n}-\alpha f_{n}\right)$ are norm decreasing and weakly measurable in $\alpha$ so the same is true of their unique extensions to $L_{2}\left(P_{x}\right)$ which we also denote by $V(\alpha)$. The operators $J(\lambda)$ defined for $\lambda>0$ by: $J(\lambda)=\int_{0}^{\infty} e^{-\lambda \alpha} V(\alpha) d \alpha$ satisfy $\|J(\lambda)\| \leqq 1 / \lambda$ and $\lim J_{n}(\lambda) \pi_{n} \phi=\lim J(\lambda) \pi_{n} \phi=J(\lambda) \phi$ for all bounded $\phi$ and hence for all $\phi$ in $L_{2}\left(P_{x}\right)$. According to a theorem of Trotter [7, Theorem 5.1, p. 900], if we can show

(i) $J(\lambda)-J(\mu)=(\mu-\lambda) J(\lambda) J(\mu)$,

(ii) $\left\|\lambda^{m} J^{m}(\lambda) \leqq\right\| M$ and

(iii) $\lim _{\lambda \rightarrow \infty} \lambda J(\lambda)=I$,

we can conclude that $V(\alpha)$ is a semigroup with resolvent $J(\lambda)$. (ii) is immediate and (i) follows from

$$
\begin{aligned}
(J(\lambda)-J(\mu)) \phi & =\lim \left(J_{n}(\lambda)-J_{n}(\mu)\right) \pi_{n} \phi \\
& =\lim (\mu-\lambda) J_{n}(\lambda) J_{n}(\mu) \pi_{n} \phi=\lim (\mu-\lambda) J_{n}(\lambda) \pi_{n} J(\mu) \phi \\
& =(\mu-\lambda) J(\lambda) J(\mu) \phi .
\end{aligned}
$$

It will be sufficient to prove (iii) for $S_{k}$-measurable $\phi$ which are bounded and have bounded derivatives since such functions are dense in $L_{2}\left(P_{x}\right)$. For such $\phi$

$$
\begin{aligned}
\lambda J_{n}(\lambda) \phi(x)= & \int_{0}^{\infty}\left[\frac{p_{n}\left(x_{1}-\alpha f_{1}, \cdots, x_{n}-\alpha f_{n}\right)}{p_{n}\left(x_{1}, \cdots, x_{n}\right)}\right]^{1 / 2} \phi\left(x_{1}-\alpha f_{1}, \cdots, x_{k}-\alpha f_{k}\right) \frac{d\left(-e^{-\lambda \alpha}\right)}{d \alpha} d \alpha \\
= & \phi(x)-\int_{0}^{\infty} e^{-\lambda \alpha}\left[\frac{p_{n}\left(x_{1}-\alpha f_{1}, \cdots, x_{n}-\alpha f_{n}\right)}{p_{n}\left(x_{1}, \cdots, x_{n}\right)}\right]^{1 / 2} \\
& \cdot\left(\sum_{i=1}^{k} f_{i} \frac{\partial \phi}{\partial x_{i}}\left(x_{1}-\alpha f_{1}, \cdots, x_{k}-\alpha f_{k}\right)\right) d \alpha \\
& -\int_{0}^{\infty} e^{-\lambda \alpha}\left(\sum_{i=1}^{n} f_{i} \frac{\partial \sqrt{ } \frac{p_{n}}{\partial x_{i}}}{x_{i}}\right)\left(x_{1}-\alpha f_{1}, \cdots, x_{n}-\alpha f_{n}\right) \frac{\phi\left(x_{1}-\alpha f_{1}, \cdots, x_{k}-\alpha f_{k}\right)}{\sqrt{p_{n}\left(x_{1}, \cdots, x_{n}\right)} d \alpha .}
\end{aligned}
$$

Since

$$
\left\|\left(\frac{p_{n}\left(x_{1}-\alpha f_{1}, \cdots, x_{n}-\alpha f_{n}\right)}{p_{n}\left(x_{1}, \cdots, x_{n}\right)}\right)^{1 / 2}\right\|=1,
$$

the norm of the first integral is bounded by $C \lambda^{-1}$ for some $C$ independent of $n$. Also 


$$
\begin{aligned}
& \left.\frac{\left(\sum_{i=1}^{n} f_{i} \frac{\partial \sqrt{ } p_{n}}{\partial x_{i}}\left(x_{1}-\alpha f_{1}, \cdots, x_{n}-\alpha f_{n}\right)\right)}{\sqrt{ } p_{n}\left(x_{1}, \cdots, x_{n}\right)}\right|^{2} \\
& =\sum_{i=1}^{n} \sum_{j=1}^{n} f_{i} f_{j} \int \cdots \int \frac{\partial \sqrt{ } p_{n}}{\partial \xi_{i}}\left(\xi_{1}-\alpha f_{1}, \cdots, \xi_{n}-\alpha f_{n}\right) \frac{\partial \sqrt{ } p_{n}}{\partial \xi_{j}}\left(\xi_{1}-\alpha f_{1}, \cdots, \xi_{n}-\alpha f_{n}\right) d \xi_{1} \cdots d \xi_{n} \\
& =\sum_{i=1}^{n} f_{i}^{2} \int \cdots \int\left(\frac{\partial \sqrt{ } p_{n}}{\partial \xi_{i}}\right)^{2}\left(\xi_{1}, \cdots, \xi_{n}\right) d \xi_{1} \cdots d \xi_{n} \leqq K^{2} \sum_{i=1}^{\infty} f_{i}^{2}
\end{aligned}
$$

so the norm of the second integral is also bounded by $C \lambda^{-1}$ and hence $\|\lambda J(\lambda) \phi-\phi\|=\lim \left\|\lambda J_{n}(\lambda) \phi-\phi\right\| \leqq C \lambda^{-1}$ which proves (iii). Now for any $0<\alpha<1$ and $\phi$ in $L_{2}\left(P_{x}\right),\|\phi\|=\left\|u_{f}^{2} \phi\right\|=\|V(1) \phi\|=\|V(1-\alpha) V(\alpha) \phi\| \leqq$ $\|V(\alpha) \phi\| \leqq\|\phi\|$ so $V(\alpha)$ is an isometry. If $\phi$ is positive and $S_{k}$-measurable, then

$$
\begin{aligned}
\int(V(\alpha) 1)^{2} \phi\left(x_{1}, \cdots, x_{k}\right) P_{x}(d x) & =\left\|V(\alpha) \sqrt{ } \phi\left(x_{1}+\alpha f_{1}, \cdots, x_{n}+\alpha f_{n}\right)\right\|^{2} \\
& =\left\|\sqrt{ } \phi\left(x_{1}+\alpha f_{1}, \cdots, x_{n}+\alpha f_{n}\right)\right\|^{2} \\
& =\int \phi\left(x_{1}+\alpha f_{1}, \cdots, x_{n}+\alpha f_{n}\right) P_{x}(d x)
\end{aligned}
$$

so that $\alpha f$ is in $M\left(x, S_{\infty}\right)$ and $(V(\alpha) 1)^{2}=d P_{x+\alpha f} / d P_{x} .(n+\alpha) f$ is in $M\left(x, S_{\infty}\right)$ because of the semigroup property of $M\left(x, S_{\infty}\right)$.

COROLlaRY 3. If in addition to the above properties $p_{n}\left(\xi_{1}, \cdots, \xi_{n}\right)=p_{n}\left(-\xi_{1}, \cdots,-\xi_{n}\right)$, then $M\left(x, S_{\infty}\right)$ is linear.

\section{REFERENCES}

1. R. M. Blumenthal and R. K. Getoor, Sample functions of stochastic processes with stationary independent increments, J. Math. Mech. 10 (1961), 493-516.

2. U. Grenander, Stochastic processes and statistical inference, Ark. Mat. 1 (1950), No. 17, 195-277.

3. K. Jacobs, Neuere Methoden und Ergebnisse der Ergodentheorie, Ergebnisse der Mathematik und ihrer Grenzgebeite, Vol. 29, Springer, Berlin, 1960.

4. T. S. Pitcher, Likelihood ratios of Gaussian processes, Ark. Mat. 4 (1960), No. 5, 35-44.

5. L Likelihood ratios for stochastic processes related by groups of transformations, Illinois J. Math. 7 (1963), 396-414.

6. A.V.Skorohod, On the differentiability of measures which correspond to stochastic processes. I. Processes with independent increments, Theor. Probability Appl. 2 (1957), 407-431.

7. H. F. Trotter, Approximations of semigroups of operators, Pacific J. Math. 8 (1958), 887-919.

LINCOLN LABORATORY $\left({ }^{2}\right)$,

LEXINGTON, MASSACHUSETTS

(2) Operated with support from the U.S. Army, Navy, and Air Force. 